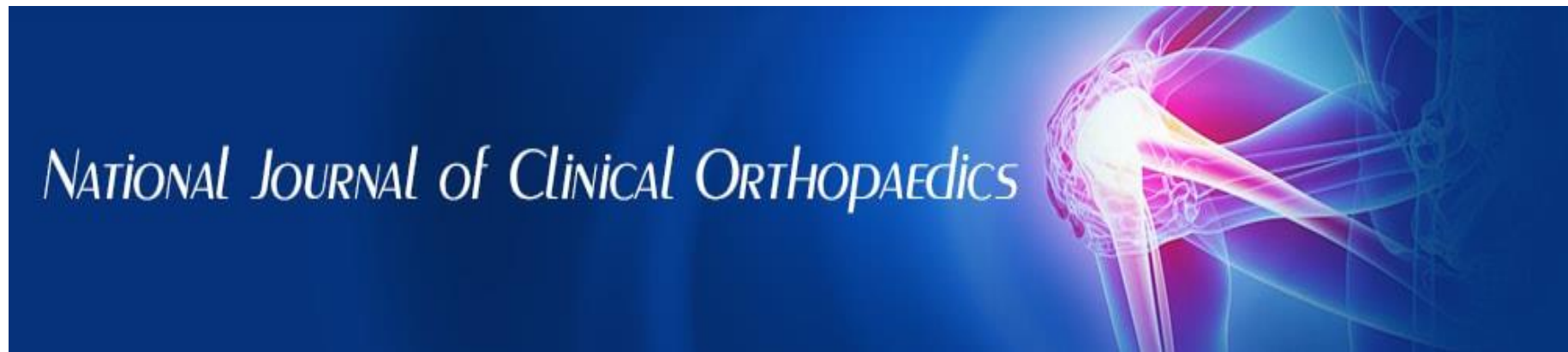

ISSN (P): 2521-3466

ISSN (E): 2521-3474

(C) Clinical Orthopaedics

www.orthoresearchjournal.com

2021; 5(2): 20-23

Received: 20-12-2020

Accepted: 25-01-2021

Ravi kumar K. Professor,

Department of Orthopaedics,

MVJ Medical College and

Research Hospital, Bangalore,

Karnataka, India

Hemanth R. Assistant

Professor, Department of

Orthopaedics, MVJ Medical

College and Research Hospital,

Bangalore, Karnataka, India

Mohammed Tanzeem P.

Junior Resident, Department of

Orthopaedics, MVJ Medical

College and Research Hospital,

Bangalore, Karnataka, India

Prajwal Sadalagi

Junior Resident, Department of

Orthopaedics, MVJ Medical

College and Research Hospital,

Bangalore, Karnataka, India
Corresponding Author:

Hemanth R. Assistant

Professor, Department of

Orthopaedics, MVJ Medical

college and Research Hospital,

Bangalore, Karnataka, India

\section{Functional outcome of Cemented Modular bipolar Hemiarthroplasty in Fracture Neck of Femur}

\author{
Ravi Kumar K, Hemanth R, Mohammed Tanzeem P, Prajwal Sadalagi
}

DOI: https://doi.org/10.33545/orthor.2021.v5.i2a.277

Abstract

Introduction: Intracapsular femoral neck fractures account for about 50\% of all hip fractures. With increase in life expectancy, the incidence is increasing. Popular treatment is replacement of femoral head by bipolar prosthesis.

Materials \& methods: This study was done to assess the functional outcome of cemented bipolar hemiarthroplasty. Patients aged 50-80 years with intracapsular femoral neck fractures reporting to the orthopaedic department of the institute were considered for the study. 30 such patients were selected. All were operated by cemented modular bipolar hemiarthroplasty. It was a retrospective as well as prospective study. Regular clinical and radiological follow up was done for a minimum period of 6 months. Results were assessed using Harris Hip score.

Results: Out of 30 patients assessed, 17 were females and 13 were males with average age was 68 years. At 6 months of follow up, out of 30 patients, excellent results were observed in $70 \%$ cases, good in $20 \%$ cases, average in $10 \%$ cases and poor in $0 \%$ cases.

Conclusion: Most patients had good to excellent functional results post cemented modular bipolar hemiarthroplasty. Hence, Cemented Modular Bipolar Hemiarthroplasty is a safe and effective treatment modality in treating femoral neck fractures in middle aged to elderly individuals.

Keywords: Femur neck fracture, Hemiarthroplasty, Modular Bipolar, Harris hip score

\section{Introduction}

Femoral neck fractures are one of the most common fractures of all hip fractures. It is seen most commonly in elderly age group which results in mortality and morbidity of patients. Most patients gives history of low energy trauma in the form of slip and fall. In few cases there could be no history of trauma and the injury is due to pathological fractures, usually due to osteoporosis, especially in elderly. With increase in life expectancy, incidence of femoral neck fractures are also increasing ${ }^{[1,2]}$.

Femoral neck fractures have presented great challenges to orthopaedic surgeons, most of the patient are associated with co-morbidities like hypertension and diabetes which leads to increased morbidity and mortality. Hence early mobilization following surgery is required in femoral neck fractures ${ }^{[3]}$. The treatment options for the fracture of the femoral neck are internal fixation, unipolar or bipolar arthroplasty or total hip arthroplasty, each having advantage and disadvantage. However, the best method of treatment still remains controversial and it has been referred to as unsolved fractures by Dickson $(1953)^{[2,4]}$.

Poor mechanical properties and osteoporosis in the elderly do not provide adequate support for the placement of screws which may eventually result in an early mechanical failure, leading to collapse, It may also lead to the migration of the femoral head into the varus and retroversion, thus causing shortening and a decrease in abductor muscle lever arm, leading to limping. It may also end up in nonunion, Avascular necrosis because of poor healing potential. Functional disabilities can also arise as a complication of the internal fixation resulting from the implant being cut out from the femoral head. Hence in elderly population replacement is the preferred modality $[2,5,6]$

Hemiarthroplasty involves replacing the femoral head with prosthesis while maintaining the acetabulum and acetabular cartilage and Total hip replacement is preferred when acetabular changes are present ${ }^{[7]}$. The aim of Hemiarthroplasty is early rehabilitation of patients with 
various co-morbidities. Out of all the treatment options available, Hemiarthroplasty is the most common treatment for displaced femoral neck fractures especially with the cemented prosthesis, which gives high success rate in elderly age group and is associated with better functional outcome and fewer reoperations ${ }^{[6,8]}$.

Four to five decades back, Single piece unipolar metal prosthesis was introduced by Thomson in 1954 and By Austin Moore In 1957 to replace femoral head as a treatment of femoral neck fractures [7, 9]. However the complications with unipolar prosthesis were Acetabular erosions and stem loosening giving rise to pain ${ }^{[10,11]}$.

Bateman in 1974 Introduced Bipolar prosthesis which had mobile head element and had additional head surface to allow movement with in the acetabulum, which led to reduce wear of acetabular surface resulting in reduced incidence of pain and acetabular protrusion due to presence of motion between metal head and Polyethylene socket (Inner bearing) and between metallic head and acetabulum (outer bearing) ${ }^{[12]}$.

In recent time, bipolar prosthesis with cement is the best option which helps the patient to recover early and become more active especially modular bipolar prosthesis with cement will give patient an active life. Modularity has the advantage of various sizes in the prosthesis which helps in maintaining limb length offset and soft tissue tension and helps in achieving identical anatomy and biomechanics as that of a hip joint $[13,14,15,16]$.

This study was done to evaluate the functional outcome of cemented modular bipolar hemiarthroplasty in femoral neck fractures by Harris Hip score.

Materials and methods: This study was conducted in the Department of Orthopaedics in MVJ Medical College and Research Hospital, Bangalore. Patients reporting to OPD and Casualty having neck of femur fracture were considered for the study with the following inclusion and exclusion criteria.

Inclusion criteria:

Age: 50 to 80 years.

Acute fracture of less than 2 weeks.

Patient surgically fit.

\section{Exclusion criteria:}

Patients below 50 yrs and above 80 years.

Pathological Fracture, other than osteoporosis.

Other associated fractures.

Fracture duration more than 2 week old.

osteoarthritis in the same hip.

30 patients were included in the study, aged between 50 to 80 years who met inclusion and exclusion criteria, having neck of femur fracture. All patients were admitted with skin traction using $2 \mathrm{~kg}$ weight and initial treatment was given. Primary evaluation was done using $X$ ray and other investigations. Systemic evaluation was done for surgical fitness and Pre anaesthetic evaluation was done after Optimization.

All patients were operated under spinal anaesthesia or combined epidural. All patients were operated using Moore's posterior approach. Fracture site was exposed, head extracted, Size measured. Neck prepared by doing osteotomy at $1 \mathrm{~cm}$ from lesser trochanter. Femoral canal prepared. Trail implant with optimal neck length was determined. Trial prosthesis were replaced by sterile prosthesis. Cemented modular bipolar prosthesis was placed after ensuring correct length, offset and soft tissue tension. Stability and range of movements checked on table. Capsule was sutured and wound was closed in layers with drain in place.

Post operatively, patient was kept with both limbs in abduction using abduction pillow. Static quadriceps and ankle exercises were started from post day 1 . Post-op day 2 drain was removed and patients were allowed to sit up at the edge of the bed. Patients were mobilized with partial weight bearing with walker support on $3^{\text {rd }}$ post-operative day, and full weight bearing was started after 2 weeks. Suture removal was done after $14^{\text {th }}$ postoperative day. Patients were instructed not to sit crosslegged or squat. Clinical and Radiological follows up were done at 3 weeks, 6 weeks, 3 months and 6 months. All patients were followed up for functional outcome using Harris hip score. According to Harris hip score, out of total 100 score, less than 70 score was considered poor, 70-80 average, 80-90 Good, More than 90 excellent.

\section{Results}

All 30 cases were followed up to minimum of 6 months duration, clinical and radiological outcome were evaluated both prospective and retrospectively. No cases were lost during the followup period.

In this study of 30 patients with femoral neck fractures, average age of patients was 68 Years, left side involved in 17 and right side is 13 , female-male ratio is $1.3: 1$. The most common mode of injury is slip and fall (27 patients) remaining had road traffic accident ( 3 patients). In the study, there were 8 subcapital, 18 Transcervical and 4 Basicervical fractures of femoral neck.

Out of 30 patient 16 patients had co morbid condition, 6 patient had Hypertension, and 10 had Diabetes mellitus.

The average duration of surgery was 60 minutes (range 40-80) with average blood loss of $200 \mathrm{ml}$ (range $80-440 \mathrm{ml}$ ). Blood transfusion is done in 2 patients pre operatively and 4 patients post operatively. Mean duration of stay in hospital is 7 days (510 days).

Out of 30 cases, at the end of 6 months follow up duration, there were 2 cases of limb length discrepancy in the form of shortening of less than $1 \mathrm{~cm}, 1$ case of chronic hip pain. There were no cases of infection, no cases of hip dislocation, There was no evidence of Stem subsidence, Heterotropic Ossification, Sciatic nerve palsy in any of the patients during the follow up period. Patients were followed up clinically, radiologically and mean Harris hip score was done at the end of 3 weeks, 6 weeks, 3 months and 6months. At 6 months of follow up, out of 30 patients, excellent results were observed in $70 \%$ cases, good in $20 \%$ cases, average in $10 \%$ cases and poor in $0 \%$ cases.

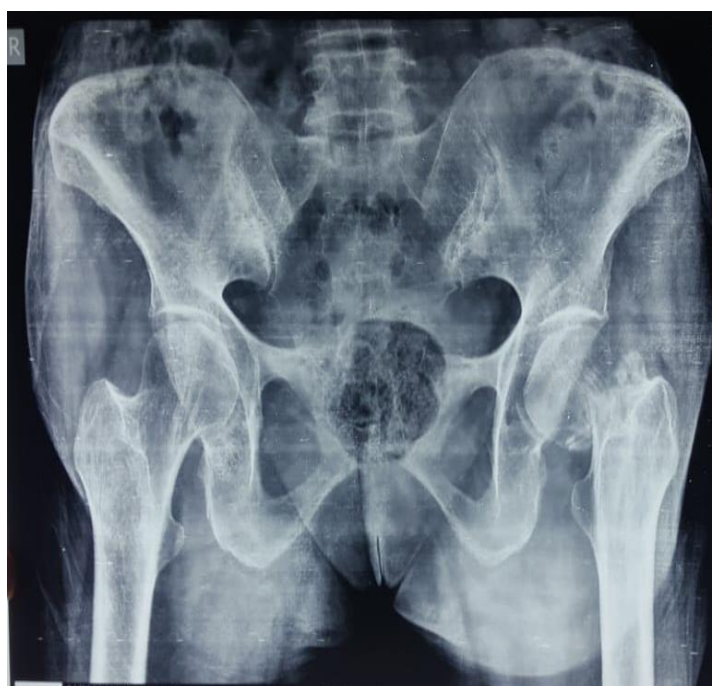




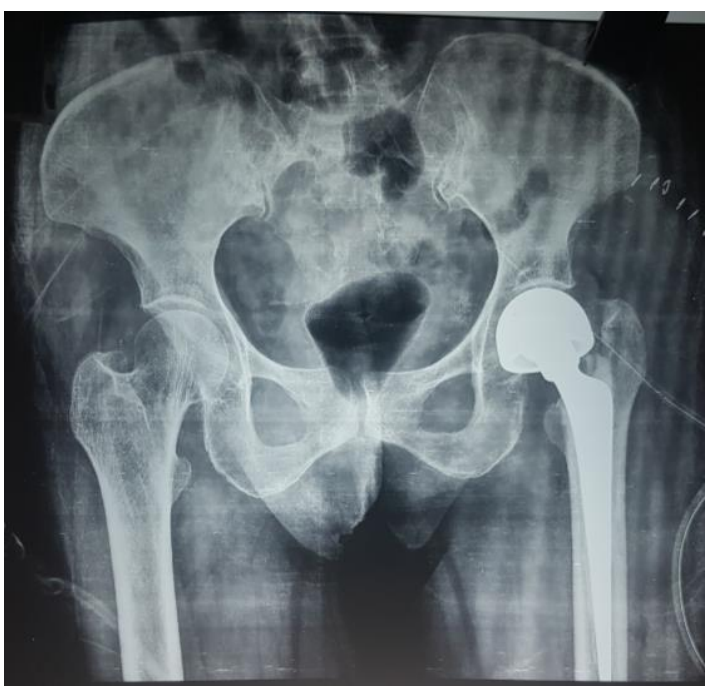

Fig: 1 and 2

Pre operative and Post operative X ray of patient with subcapital fracture neck of femur, operated with cemented modular bipolar hemiarthroplasty. This patient had shortening less than $1 \mathrm{~cm}$.

\section{Discussion:}

Fracture neck of femur is one of the most common fractures in the elderly population resulting from a trivial trauma. Hip fractures are one of the most important and challenging orthopaedic injuries. Several treatment modalities have been used for treating these injuries [2, 6]. Hemiarthroplasty with bipolar prosthesis is most commonly preferred treatment in femoral neck fractures as internal fixation with Osteosynthesis ended up with higher rates of re-operation $\left[\begin{array}{ll}17, & 18,19]\end{array}\right.$ Hemiarthroplasty helps in faster recovery, pain relief and early rehabilitation ${ }^{[8,11]}$.

Initially Bipolar prosthesis were of Non Modular design which was followed by

Modular design of the prosthesis which allowed neck length adjustment with interchangeable stems which helps in maintaining limb length, femoral offset and soft tissue balancing. Further conversion to a total hip replacement was easier with modular prosthesis as only acetabular component needs to be added ${ }^{[7,14,16]}$.

The mean age of cases with femoral neck fracture in our study was 68 Years ranging from 50 years to 80 years, which was comparable to other studies. Rakshith Kumar et al. In their study found an average age of 65years in 20 patients with fracture neck of femur treated with bipolar hemiarthroplasty.

Hanu Tej Adapureddi et al. Reported an average age of 65 years in 50 patients with femoral neck fracture.

The gender distribution of the cases was 17 females and 13 males amounting for $57 \%$ and $43 \%$ respectively, which is comparable to other studies. Rakshith Kumar et al. Reported $60 \%$ of females and $40 \%$ of males in a study of 20 patient undergoing bipolar hemiarthroplasty fracture neck of femur. Hanu Tej Adapureddy et al reported 62\% Females and 38\% males out of 50 patients.

In our study, Mode of injury was due to trivial trauma in the form of Tripping and slip and fall (90\%), which can be comparable to the study by Ravi M Doddamani (2016) ${ }^{[14]}$ who reported $92 \%$. Rest are due to Road traffic accidents (10\%).
In our present study, out of 30 cases, right side involvement was in 13 patients accounting to $43 \%$ and left side involvement was in 17 patients accounting to $57 \%$ of the cases. Rakshith kumar et al. In their study, reported slight predominance of left sided fracture when compared to right. Which was comparable to our study.

Hanu tej Asapureddy et al in their study, Left side involvement is seen in $65 \%$ of the patients and right side involvement was $34 \%$ of the total patients which was comparable to our study.

In the present study the most common anatomical fracture pattern was transcervical which is seen in $60 \%$ of the cases, followed by Subcapital in $27 \%$ of the cases and basicervical in $13 \%$ of the cases. Rakshith Kumar et al. In their study found $80 \%$ of patient with transcervical fracture, $15 \%$ of patients with basicervical fracture and 5\% of patients with subcapital fracture. Hanu Tej Adaoureddy et al. in their study $80 \%$ cases were transcervical, $16 \%$ were Basicervical and $4 \%$ subcapital fractures which were comparable with our study.

At the final followup of 6 months, the mean Harris hip score was 88 points with excellent results were observed in $70 \%$ cases, good in $20 \%$ cases, fair in $10 \%$ cases and poor in $0 \%$ cases. Rakshith kumar et al in their study $30 \%$ had achieved excellent result, $45 \%$ had achieved good result, $10 \%$ achieved fair result and $15 \%$ achieved poor result.

Hanu tej Adapureddy et al in their study, 35\% achieved excellent results, $45 \%$ achieved good results, $10 \%$ achieved fair result and $10 \%$ achieved poor result.

In the present study most of the patient at the end of 6months were able to carryout the activities of daily living by themselves and required minimum support by others. $90 \%$ of patients were returned to previous functional status. Similar results were reported by Rakshith kumar et al. who reported $85 \%$ patients return to the functional level that they had before the fracture and Hanu Tej Adapureddy et al reported $80 \%$ patients return to the functional level that of pre fracture.

The complications that occurred were a total of 3 in 30 patients accounting for $10 \%$, Similar complication were reported by Hanu Tej Adapureddy et al. In their study had superficial infection in the form of wound dehiscence in 3 patient.

Limb length discrepancy is seen in 2 cases out of 30 cases $(6.6 \%)$. Shortening was seen in 2 cases, of less than $1 \mathrm{~cm}$, inspite of checking it intraoperatively. There was no lengthening, Similar result were reported by Hanu Tej Adapureddy et al. who reported limb length discrepancy seen in 2 patients in the form of limb shortening by $1-1.5 \mathrm{~cm}$ managed by shoe rise.

In our study, Limb length discrepancy was seen in 2 patient in the form of shortening of less than $1 \mathrm{~cm}$ which did not require any treatment. 1 patient had mild Chronic hip pain probably due to preexisting mild hip arthritis which was managed conservatively by analgesics.

Average results in the study were probably because of age related preoperative limitations in mobility and due to articular cartilage degeneration in acetabulum.

Limitation of our study was that Harris Hip score could not be used to compare pre and post operative outcomes as ambulatory status of the patient was not known before surgery. All average results of Harris hip score may not be attributable to surgery.

\section{Conclusion}

In our study, Cemented Modular Bipolar Hemiarthroplaty as a treatment modality demonstrated good to excellent functional 
results with less complications in patients having femoral neck fractures. Hence, we conclude that Cemented Modular Bipolar Hemiarthroplaty is a safe and effective treatment modality.

\section{References:}

1. Charles M, Court-Brown, James D Heckman et al. 8th Edition, Rockwood and Green's Fracture in Adults.

2. Swiontowski MF Intracapsular fractures of the hip. J Bone Joint Surg Am 1994;76:129-138.

3. S Terry Canale, James H Beaty, Frederick M Azar, 13th Edition, Campbell's operative orthopaedics.

4. Dickson JA The unsolvedfracture jbjs Surg 1984:35:805822.

5. DK Dedrick, JR Mackenzie, RE Burneg. Complications of femoral neck fractures in young adults. J trauma. 1986;26(10):932-7.

6. Jilian Kazley, Kaushik Bagchi, Femoral neck fracture - Stat Pearls-NCBI Bookshelf Jan 2020 May.

7. GS Kulkarni, Sushruth Babuhlkar $3^{\text {rd }}$ edition Texbook of Orthopaedics and Trauma.

8. SC Jonas, R Shah, N AL-Hadithy, MR Norton, SA Sexton, RG Middleton. Displaced intracapsular neck of femur fracture in elderly: Bipolar hemiarthroplasty may be the treatment of choice: A case control study. Injury. 2015;49(10):1988-91.

9. Clayer M, Bruckner J. The outcome of Austin moore hemiarthroplasty for fracture of femoral neck. Am J Orthop 1997:26:681-4.

10. Zofka P. Bipolar hip hemiarthroplasty. Acta Chir Orthop Traumatol Cech 2007;74(2):99-104.

11. Inngul C, Hedbeck CJ et al. Unipolar hemiarthroplasty versus bipolar hemiarthroplasty in patients with displaced femoral neck fractures: a four-year follow-up of a randomised controlled trial. Int Orthop 2013;37(12):245764.

12. Bateman JE. Single-assembly total hip prosthesis: a preliminary report. Orthop Dig 1974;2:15.

13. Rakshith Kumar K. Maheshwarappa Prospective study of hemiarthroplasty for intercapsular fracture neck of femur using modular bipolar prosthesis. IJOS 2018:4(4):92-95.

14. Ravi m Daddimani, Vishwanath M Patil et al. Results of fracture neck of femur treated with modular bipolar hemiarthroplasty in elderly our experience in 70 cases International journal of orthopaedics traumatology and surgical sciences 2016;2(1):197-202.

15. Hanu Tej Adapureddi, SB Kamareddy, Anand Kumar et al. Prospective study of Management of fracture neck of femur by Hemiarthroplasty with cemented bipolar. Journal of Evolution of Medical and dental sciences 2015;4(98)07: page 16309-16314.

16. Zahid Bashir, Zubair Younis Ringshawl et al. Functional outcome of uncemented modular bipolar hemiarthroplasty using modified harris hip score for fractures of femoral neck in elderly patients JMSCR 2020, 8(9).

17. Rogmark C, Calsson A, Johnell O et al. A prospective randomized trial of internal fixation versus arthroplasty for displaced fractures of neck of the femur; Functional outcome for 450 patients at two years. J Bone Joint Sug (Br) 2002;84:183-8.

18. Parker mj, Khan RJ, Crawford J, Pryor GA. Hemiarthroplasty versus internal fixation for displaced intracapsular hip fractures in elderly A randomized trial of 455 patients. J Bone Joint Surg Br 2002;84:1150-5. 